\title{
Effects of JPEG Compression on Reading Optical Watermarking Embedded by Using Color-Difference Modulation
}

\author{
Kazutake Uehira, Hiroshi Unno \\ Department of Information Network and Communication, Kanagawa Institute of Technology, Atsugi, Japan \\ Email: Uehira@nw.kanagawa-it.ac.jp
}

How to cite this paper: Uehira, K. and Unno, H. (2018) Effects of JPEG Compression on Reading Optical Watermarking Embedded by Using Color-Difference Modulation. Journal of Computer and Communications, 6, 56-64.

https://doi.org/10.4236/jcc.2018.61006

Received: September 18, 2017 Accepted: December 26, 2017 Published: December 29, 2017

\begin{abstract}
In this paper, we evaluate the readability of optically written watermarking from an image compressed by JPEG. We previously proposed an optical watermarking technique that can protect the portrait rights of real objects. It produces a watermarking pattern in the illumination light by modulating color differences. The illumination light that contains such watermarking is projected onto an object. An image of the object taken by a camera contains the same watermarking, which can be extracted by image processing. Therefore, this technique can protect the portrait rights of real objects. We conducted simulations of capturing an object image illuminated by watermarked light, compressing it by JPEG, and reading embedded information from the decoded image. The simulation results showed that the accuracy in reading out embedded information decreases when captured images are compressed. However, for medium-level or low compression rates 100\% accuracy can be expected by using the error correction technique.
\end{abstract}

\section{Keywords}

Digital Watermarking, Information Embedding, Portrait Rights

\section{Introduction}

The distribution of digital images is increasing as the Internet becomes broadband and cloud services become popular. This has increased the importance of protecting the copyright of digital images distributed on the Internet because digital images can easily be copied in a way such that they are exactly like the original.

Digital watermarking is an effective way of protecting digital image copyrights 
from being illegally copied and various digital watermarking techniques for digital images have been developed [1] [2] [3] [4].

Digital watermarking has also been used in printed images, where digital watermarking is embedded in the digital data before the images are printed [5] [6] [7]. This is to prevent the images from being copied from printed images by digital cameras or scanners.

However, conventional digital watermarking rests on the premise that people who want to protect the copyrights of their digital content, i.e., content creators or content providers, have the original digital data and they can embed watermarking in the original digital data by digital processing.

However, this premise does not always hold. For example, it does not hold in the case that a person took a photo of a painting at a museum with a digital camera. Since recent digital cameras are highly advanced, captured images have very high levels of quality and if the painting is invaluable as a portrait, the captured image of the painting also has high value. Therefore, the portrait rights of such paintings should be protected. However, images captured with digital cameras do not have watermarking in this case because they have been captured by visitors to museums who are not interested in protecting portrait rights.

We previously proposed a technique that could prevent the copyright or portrait rights of images of real objects captured with a camera [8] [9]. It used illumination that contained invisible watermarking. As the illumination contained the watermarking, the captured images of objects that were illuminated by such illumination also contained watermarking. We demonstrated the feasibility of this technique producing watermarking by spatially modulating the brightness of the illumination. Moreover, we produced watermarking by modulating color differences in illumination and demonstrated that readability and invisibility of the embedded information in this method were superior to the brightness modulation method [10].

In this paper, we describe the effects of image compression by JPEG on the readability of the embedded information. The motivation of this study was that image data captured with a digital camera are usually compressed and stored in the camera's memory and that the image compression may cause the embedded watermarking data to change or disappear. We conducted simulations to evaluate the effects of the image compression and in this paper present the obtained results. This template, created in MS Word 2007, provides authors with most of the formatting specifications needed for preparing electronic versions of their papers. All standard paper components have been specified for three reasons: 1) ease of use when formatting individual papers, 2) automatic compliance to electronic requirements that facilitate the concurrent or later production of electronic products, and 3) conformity of style throughout a journal paper. Margins, column widths, line spacing, and type styles are built-in; examples of the type styles are provided throughout this document and are identified in italic type, within parentheses, following the example. Some components, such as multi-leveled equations, graphics, and tables are not prescribed, although the various 
table text styles are provided. The formatter will need to create these components, incorporating the applicable criteria that follow.

\section{Embedding Watermarking in Illumination by Modulating Color Differences}

Figure 1 outlines the basic concept underlying our watermarking technique using illumination light to embed a watermark. A real object is illuminated by projected light that contains an invisible watermark. A photograph taken of the object illuminated in this way would also contain the same watermark as that in the illumination light. The watermark can be extracted in the same way as that used in conventional digital watermarking techniques for digital images.

There are various ways of producing optical watermarking; Figure 2 illustrates one of them. YCbCr (Y: luminance, $\mathrm{Cb}$ : chroma-blue, Cr: chroma-red) signal is used and among them the $\mathrm{Cb}$ component (a color difference signal) is modulated to produce the watermarking. The basic procedure for producing the watermarking is the same as that in we reported in our previous study, where we produced watermarking by modulating the brightness [9]. First, the whole image area that corresponds to the illumination area is divided into numerous blocks as shown in the figure. The initial data of the $\mathrm{Cb}$ component is produced as the frequency domain data for each block. Each block only has the highest frequency component (HC) in both the $\mathrm{x}$ and $\mathrm{y}$ directions. The absolute value of HCs is the magnitude of modulation. We express one-bit binary data as being embedded by the sign of the HCs, i.e., if an HC is positive, it is expressed as "1" and if it is negative, it is expressed as " 0 ".

After setting HCs for all blocks, the frequency domain data for each block is converted into block image data in the space domain by inverse discrete cosine transformation (i-DCT), and all block images are combined into one image. The

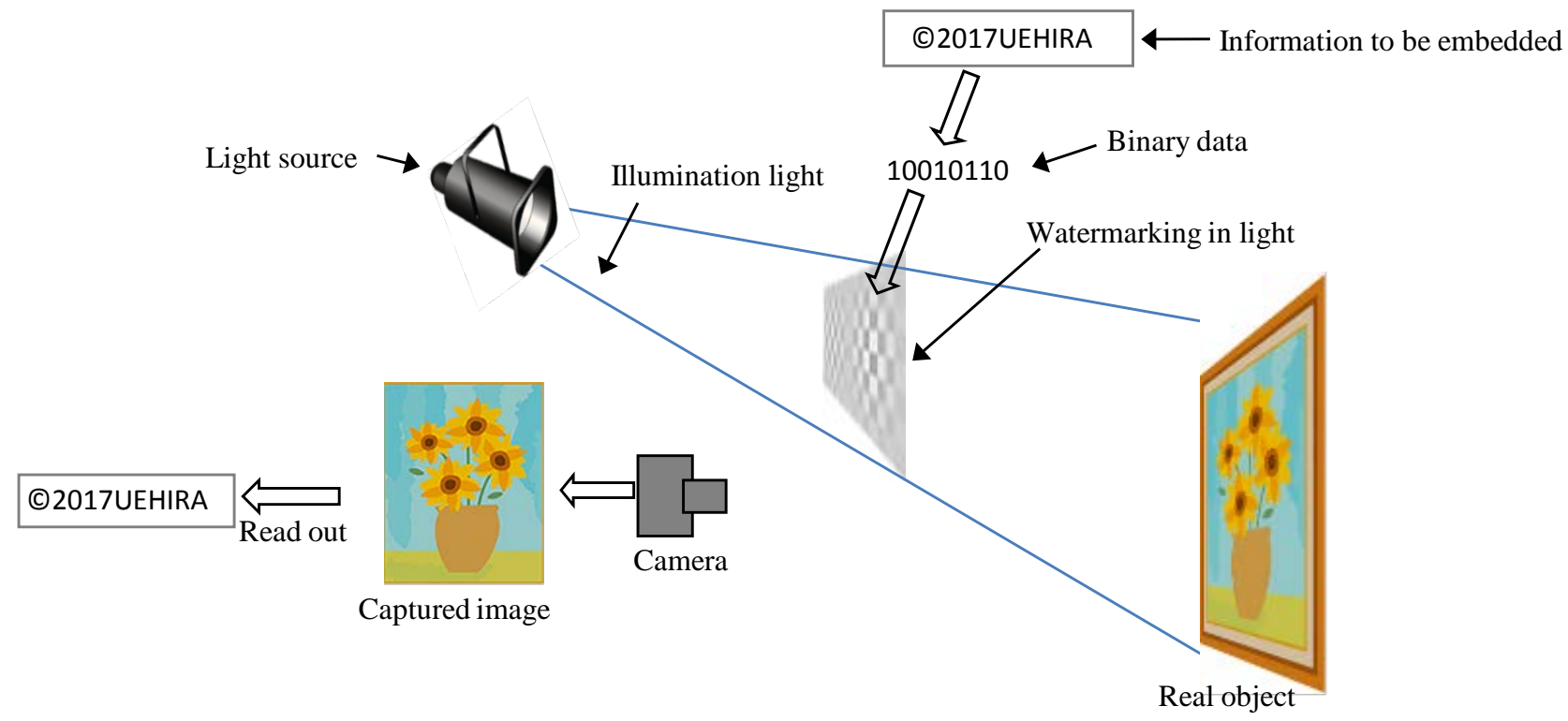

Figure 1. Basic concept underlying proposed technique. 


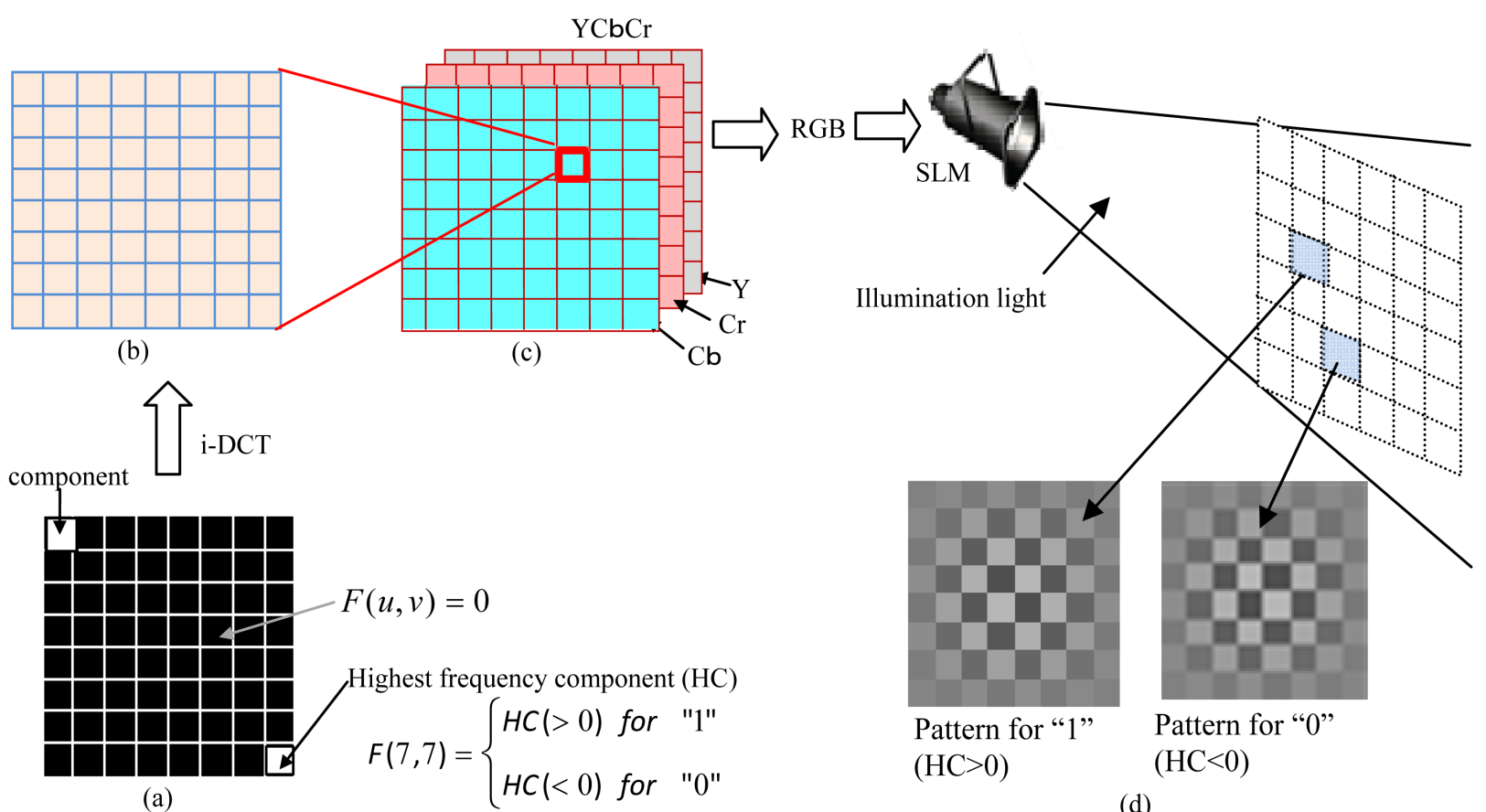

Figure 2. Procedure for producing optical watermarking. (a) Block data of $\mathrm{Cb}$ in frequency; (b) Block image data in space domain; (c) Whole image data; (d) Watermarking patterns. These two patterns have opposite phases.

$Y$ is a set constant value in the whole image area. This value gives averaged brightness. The $\mathrm{Cr}$ component is set to zero in the whole image area. Finally, the $\mathrm{YCbCr}$ signal is converted to an RGB signal, input to a space light modulator (SLM), and changed to illumination light that illuminates real objects such as paintings. We were able to use a commercial projector as an SLM for this purpose. Figure 2(d) shows the two watermarking patterns in the light; these patterns are for the " 1 " and " 0 " of binary data. Both are highest-frequency patterns but have opposite phases.

The captured image of the real object illuminated by the illumination light also has a high-frequency pattern in the $\mathrm{Cb}$ component image; that is, the captured image also contains watermarking.

The watermarking pattern in the light and in the captured image cannot be seen by the human visual system because it is modulated at the highest frequency and the amplitude of modulation is small.

Figure 3 indicates the procedure from capturing the image to reading out the embedded watermarking. In practical use, captured images are usually compressed before being stored in the camera's memory. However, this process decreases the high frequency component and may decrease the accuracy in reading out the embedded watermarking.

After the compressed image is decoded it is converted into a $\mathrm{YCbCr}$ signal, and then the $\mathrm{Cb}$ component is divided into blocks. The pixel data in each $\mathrm{Cb}$ component block is then converted into data in the frequency domain by discrete cosine transformation (DCT). Finally, the embedded data are read out by 


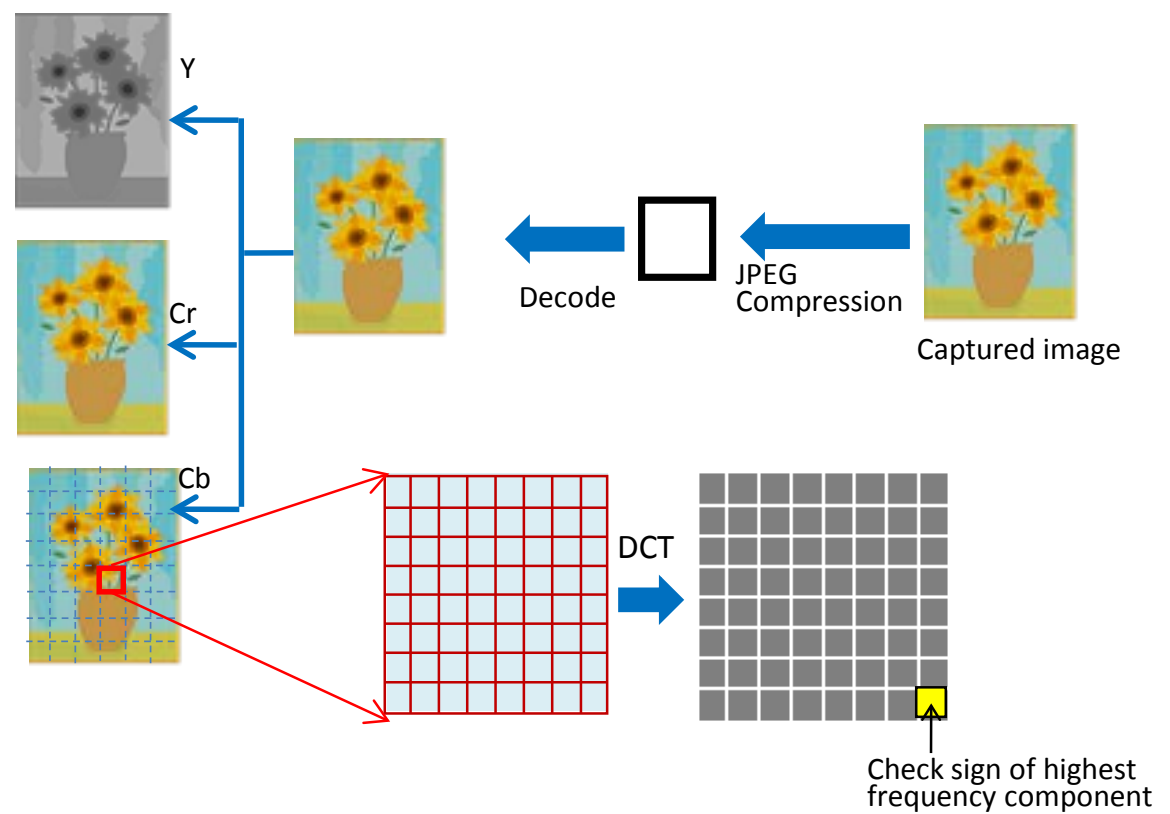

Figure 3. Procedure from capturing image to reading out embedded watermarking.

checking the sign of the frequency component in which binary data are embedded for each block.

\section{Simulation}

We evaluated the effect of image compression on accuracy in reading out embedded watermarking by simulating the procedure of capturing object images, compressing the images, and reading out the embedded information. We used JPEG to compress the images.

\subsection{Simulating Object Image Capturing}

The image data, $I(x, y)$, of an object captured with a camera is proportional to the brightness of the object surface. The brightness, $B(x, y)$, is obtained as a product of the reflectance of the object surface, $R(x, y)$, and the luminance of the projected light at the object surface, $L(x, y)$, as

$$
B(x, y)=R(x, y)\left\{L(x, y)+L_{0}\right\}
$$

where $L_{0}$ is bias luminance such as that produced by room light.

As objects we used standard images that had $512 \times 512$ pixels, as shown in Figure 4. That is, we used RGB pixel values of standard images as the reflectance of the object surface, $\mathrm{R}(x, y)$, as shown in Equation (1). In order to execute multiplication on a pixel-by-pixel basis in the equation, we set the number of pixels in the pattern projected on the object to also be $512 \times 512$ pixels.

We first generated the data of the $\mathrm{Cb}$ component in the frequency domain for $L(x, y)$ in Equation (1), as shown in Figure 2(a). The data were generated for each block. The signs of the highest frequency component (HC) for each block were determined depending on whether " 1 " or " 0 " was to be embedded in that 


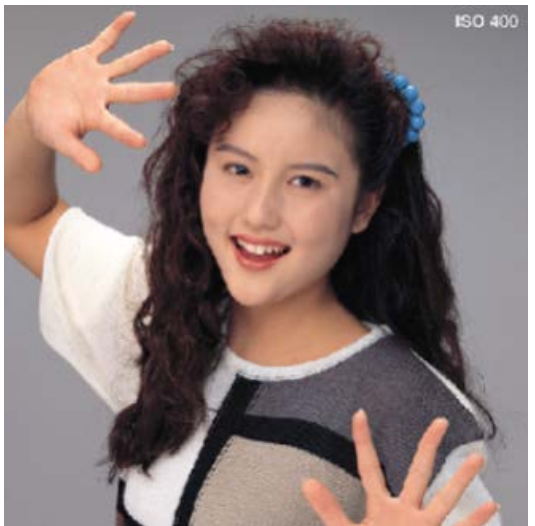

(a)

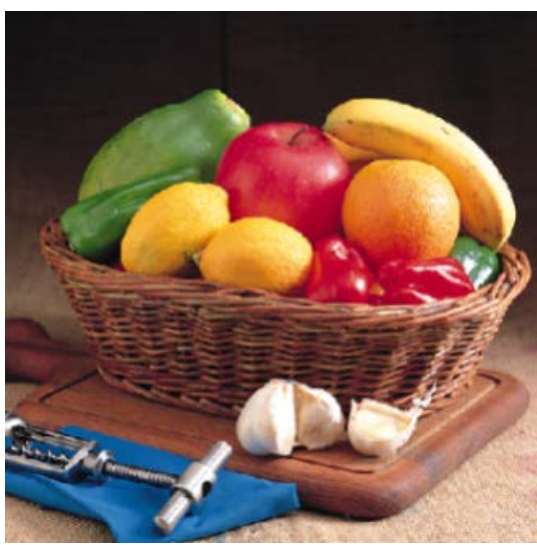

(c)

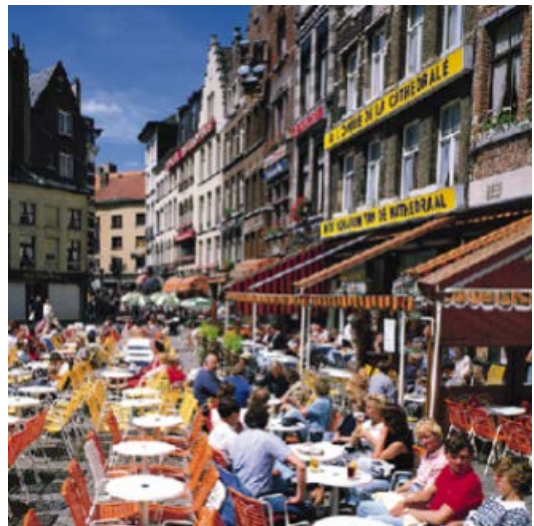

(b)

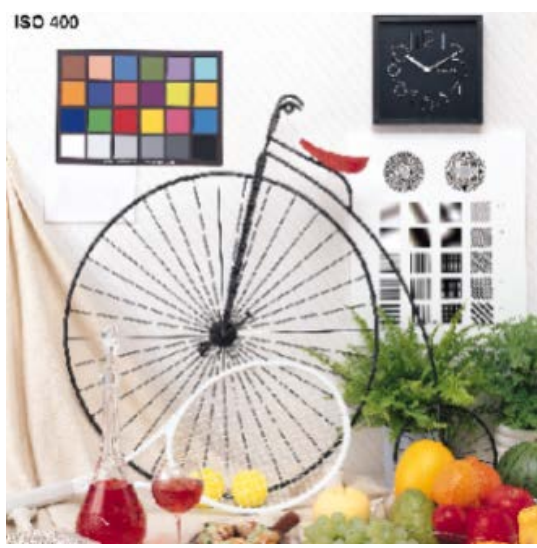

(d)

Figure 4. Images used as objects in simulation. (a) Image A; (b) Image B; (c) Image C; (d) Image D.

block. The same " 1 " and "0" numbers were randomly embedded. The magnitude of $\mathrm{HC}$ in the original data was changed from one to 10 as an experimental parameter, while Y, Cr, and L0 were set to constant values of 200, 0 , and 40. These values were the gray levels of image data whose maximum was 255 .

The captured image, $I(x, y)$, is proportional to $B(x, y)$. However, according to sampling theory, in order for $I(x, y)$ to have the highest frequency component that $B(x, y)$ has, $I(x, y)$ needs to have twice as many pixels in the vertical and horizontal directions as $\mathrm{B}(x, y)$. Therefore, we produced $I(x, y)$ by doubling the number of $B(x, y)$ pixels vertically and horizontally. Therefore, the image had $1024 \times 1024$ pixels.

\subsection{Simulating and Decoding Image Compression by JPEG}

We used JPEG as an image compression algorithm. JPEG consists of a quantizing process and Huffman encoding. Since Huffman encoding is a reversible process, we simulated only the quantization process. In the JPEG quantization process, frequency components in block data in the frequency domain are divided by certain numerical values predetermined for each frequency component. These divisors are obtained by dividing the values in the quantization table by 
numerical values called scale factors. Table 1 indicates the quantization table used in this simulation; scale factors of 1 to 10 were used. The larger the divisor for the frequency component is, the higher the compression rate becomes. Therefore, when the scale factor is one the divisor for the frequency components and the compression rate both have maximum values. On the other hand, when the scale factor is ten, the compression rate has a minimum value. We consider that accuracy in reading out the embedded information depends on the compression rate.

\subsection{Simulation of Reading out Embedded Information}

A decoded image was divided into $4096(64 \times 64)$ blocks. For each block, $\mathrm{Cb}$ components were converted into data in the frequency domain by the DCT.

Embedded data were read out by checking the sign of the $(7,7)$ frequency component of the $\mathrm{Cb}$ for each block. The accuracy with which data was read out was evaluated on the basis of the percentage of data that was correctly read out from 4096 binary data.

\section{Results and Discussion}

Figure 5 shows the obtained simulation results on the accuracy with which the binary data was read out. As expected, they revealed that the accuracy was lower when images were compressed than when they were not. They also show that the accuracy becomes lower as the scale factor becomes smaller (i.e., as the compression rate becomes higher).

It is desirable for the accuracy to reach $100 \%$ for $\mathrm{HC}$ under 10 because at that $\mathrm{HC}$ level watermarking is almost invisible when $\mathrm{Cb}$ is modulated [10]. However, it is difficult to reach $100 \%$ when an image is compressed.

In cases where an error can be expected to occur when embedded information is read out, we can use the error correction technique. In fact we used this technique in a previous study where we modulated the luminance [8]. In doing so, we embedded one binary data at three positions far from each other. When we read the data out, we used majority decision to determine the correct data if these three data are not the same. Using this technique, we were able to obtain

Table 1. Quantization table used in experiment.

\begin{tabular}{llllllll}
\hline 16 & 11 & 10 & 16 & 24 & 40 & 51 & 61 \\
12 & 12 & 14 & 19 & 26 & 58 & 60 & 55 \\
14 & 13 & 16 & 24 & 40 & 57 & 69 & 56, \\
14 & 17 & 22 & 29 & 51 & 87 & 80 & 62, \\
18 & 22 & 37 & 56 & 68 & 109 & 103 & 77 \\
24 & 35 & 55 & 64 & 81 & 104 & 113 & 92 \\
49 & 64 & 78 & 87 & 103 & 121 & 120 & 101 \\
72 & 92 & 95 & 98 & 112 & 100 & 103 & 99 \\
\hline
\end{tabular}




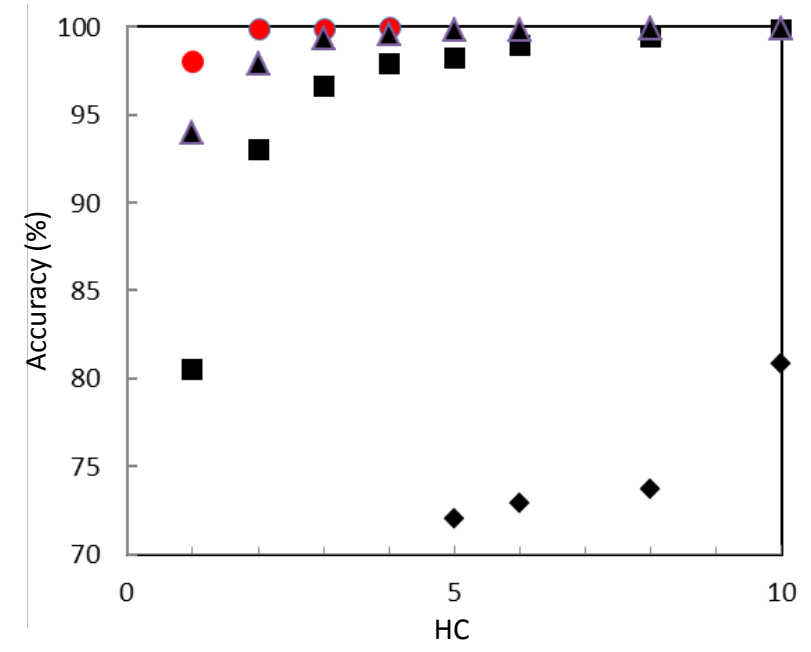

(a)

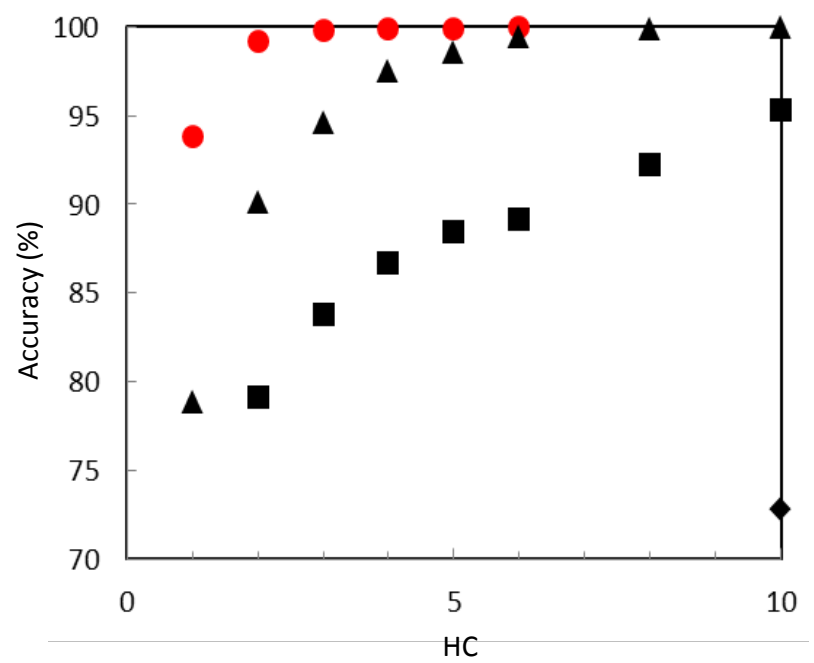

(c)

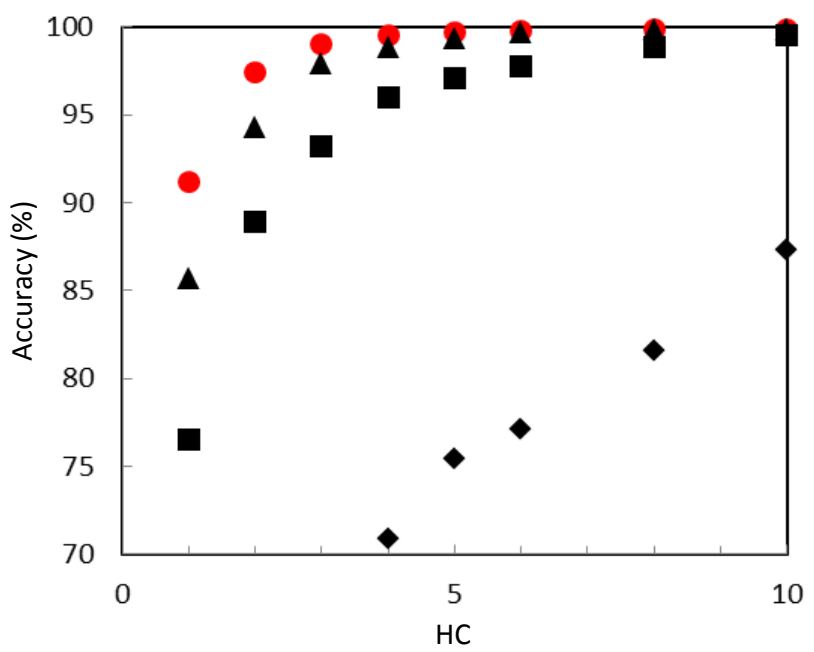

(b)

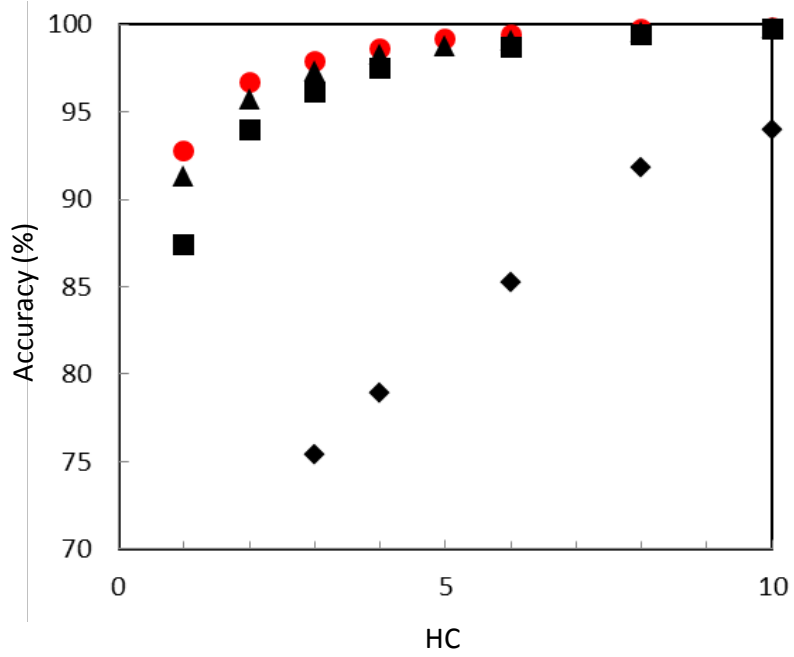

(d)

Figure 5. Accuracy obtained in reading out binary data. Accuracy indicates percentage of data correctly read out from 4096 binary

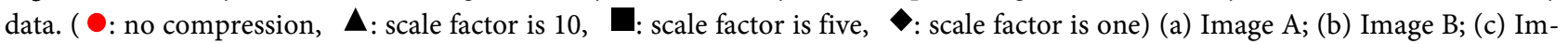
age C; (d) Image D.

$100 \%$ accuracy in reading out embedded information even if it had been around $80 \%$ when error correction was not used. Therefore, we expected that in this study $100 \%$ accuracy could be achieved by using the error correction technique except for cases when the scale factor was one. A scale factor of one results in high compression rate and poor image quality. A scale factor of five results in medium level compression rate and medium level image quality, and a scale factor of 10 results in low compression rate and high image quality. Therefore, this technique can be used for medium-level and high quality images.

\section{Conclusion}

In this study, we evaluated the accuracy of reading out optically written watermarking from a captured image compressed by JPEG. We conducted simula- 
tions of capturing an image of an object illuminated by the watermarked light, compressing it by JPEG, and reading out the embedded information from the decoded image. The simulation results showed that accuracy in reading out embedded information decreases when the captured image is compressed, but that for medium-level or low compression rates $100 \%$ accuracy can be expected by using the error correction technique.

\section{Acknowledgements}

This study was supported by the Japan Society for the Promotion of Science (JSPS) under Research Institute Grant No. 16H02820.

\section{References}

[1] Cox, I.J., Kilian, J., Leighton, F.T. and Shamoon, T. (1997) Secure Spread Spectrum Watermarking for Multimedia. IEEE Transactions on Image Processing, 6, 1673 1687. https://doi.org/10.1109/83.650120

[2] Hsu, C.-T. and Wu, J.-L. (1999) Hidden Digital Watermarks in Images. IEEE Transactions on Image Processing, 8, 58-68. https://doi.org/10.1109/83.736686

[3] Hartung, M. and Kutter, M. (1999) Multimedia Watermarking Techniques. Proceedings of IEEE, 87, 1079-1107. https://doi.org/10.1109/5.771066

[4] Langelaar, G.C., Setyawan, I. and Lagendij, R.L. (2000) Watermarking Digital Image and Video Data. IEEE Signal Processing Magazine, 17, 20-46. https://doi.org/10.1109/79.879337

[5] Mizumoto, T. and Matsui, K. (2002) Robustness Investigation of DCT Digital Watermark for Printing and Scanning. Trans. $\operatorname{IEICE}(A)$, J85-A, 451-459.

[6] Ejima, M. and Miyazaki, A. (1999) Digital Watermark Technique for Hard Copy Image. Trans. IEICE ( $A)$, J82-A, 1156-1159.

[7] Horiuchi, Y. and Muneyasu, M. (2004) Information Embedding to the Printing Images Based on DCT. Proceedings of ITC-CSCC2004, 7F3P50-1-4.

[8] Uehira, K. and Suzuki, M. (2008) Digital Watermarking Technique Using Brightness-Modulated Light. Proceedings of the IEEE ICME2008, 257-260.

[9] Ishikawa, Y., Uehira, K. and Yanaka, K. (2010) Practical Evaluation of Illumination Watermarking Technique Using Orthogonal Transforms. Journal of Display Technology, 6, 351-358. https://doi.org/10.1109/JDT.2010.2049336

[10] Uehira, K. and Unno, H. (2017) Optical Watermark Pattern Technique Using Color-Difference Modulation. Proceedings of PATTERN2017. 